\title{
Staying mindful: Lived experience and mental health care reform in Canada
}

\author{
Amanda K. Slaunwhite
}

\section{University of Victoria}

\begin{abstract}
"We get [mental health] consumer representation on our committees and boards and then snow them under with bureaucratic paper and administrivia. We burn them right out and then we cluck sadly and whisper that they weren't really up to it after all. Their medication needs adjusting, we say, and we are really so damn sympathetic." 1
\end{abstract}

\section{A Decade of Momentum}

In the past decade, mental health and mental health care have become increasingly popular issues in Canada due to provincial, federal and private sector investments in mental health promotion and treatment, and the growing visibility of the consumer and caregiver movement throughout Canada. From 2005-2012, seven provinces and one territory introduced mental health strategies, and the first national Mental Health Strategy for Canada was released by the Mental Health Commission of Canada in 2012. ${ }^{2}$ These developments have heightened the profile of mental health across Canada, and have provided momentum for new consumer-informed, privately funded public education campaigns such as Partners for Mental Health that works to reduce the stigma associated with mental health problems and accessing mental health care. ${ }^{3}$

While these were important milestones in Canada's history of mental health services, the momentum would not have been possible without the work of thousands of Canadians with lived experience. ${ }^{4}$ Canadians with lived experience include any person that has had some experience with mental health, mental illness or mental health care, including consumers of mental health services, and family members and caregivers of persons with mental health problems. To ensure the success of current and future initiatives aimed at reforming mental health services, this paper argues that it is imperative that policy development be led and informed by the perspective of persons with lived experience throughout Canada. Involving persons with lived experience in the policy development and implementation process will better ensure that policy reforms promote a consumer-focused system of care and recovery model of treatment. The ongoing involvement of persons with lived experience in health care reform can also enhance transparency and accountability in health care service management; improve the quality and effectiveness of services, and provide legitimacy and creditability to new investments or policy directions. . $^{5-7}$

\section{Conceptualizing Engagement and Barriers to Meaningful Involvement}

The perspective of persons with lived experience can be obtained though consultation, participation, and engagement in relation to direct service delivery and treatment, in addition to service system planning and policy development. ${ }^{7}$ The ladder of citizen participation has been conceptualized as a continuum. At one end of the continuum is information dissemination and consultation, where participants are given an opportunity to express their perspective, but there is no commitment to ongoing involvement or using the information gathered to develop policy or programs. ${ }^{8}$ On the opposite side of the continuum is full engagement where there is an ongoing commitment to participants to use the information gathered to develop policies or services and continually involve them in the policy development process. ${ }^{8}$ Engagement of persons with lived experience is the optimal approach for including consumers, family members, and caregivers in the policy development process because there is a clear commitment to ongoing participation that helps build trust between participants and staff. Although the engagement of persons with lived experience is the optimal approach for authentic engagement, it has not been used 
C consistently in the development of mental health strategies throughout Canada. Most provincial and territorial mental health strategies have used online surveys, town-halls, or discussion groups to gather feedback from persons with lived experience on particular policies, programs or approaches under consideration with no ongoing commitment to continuous engagement. Planning committees composed of persons with lived experience with ongoing terms of reference have been less widely used to inform policy development; however, those that have been created have been influential in shaping the focus of policy documents such as Toward Recovery and Wellbeing: A Framework for a Mental Health Strategy for Canada. ${ }^{9}$

There are several individual and organizational level factors that inhibit authentic engagement that must be addressed if persons with lived experience are to be meaningfully involved in the development and implementation of mental health care reform in Canada. Persons with lived experience must individually balance competing demands on their time, such as caregiving responsibilities, as well as feelings of apathy related to participation, and a lack of information on engagement opportunities and research and policy development techniques. ${ }^{7,8}$ Organizations must address significant barriers that negatively influence engagement efforts such as stigma towards persons with mental health problems; power imbalances among staff and participants; unhealthy work environments and work cultures, and financial and resource limitations., ${ }^{5,7}$ The redistribution of power in the policy development process and competing political demands must also be addressed within organizations to enhance the success of engagement initiatives. Within engagement exercises themselves, the barrier of scientific knowledge being privileged over lived experience of mental health issues must also be actively acknowledged and managed in order to create an equitable foundation for all knowledge to be valued in the policy development process. ${ }^{5}$

\section{Enhancing Engagement in Mental Health Care Reform}

Enhancing the engagement of persons with lived experience in mental health care reform requires that governments, regional health authorities, and others involved in the delivery of mental health care, make a commitment to inclusive planning, policy development, and priority setting in partnership with consumers, caregivers and family members. These efforts would be strengthened by encouraging the recruitment of traditionally underrepresented populations such as Aboriginal peoples, youth, young caregivers, immigrants, refugees and ethno-cultural groups on planning committees. Similarly, promoting the employment of persons with lived experience in mental health related organizations and government departments may also assist these efforts. ${ }^{6,8,10}$ The creation of a national policy and practice collaborative focused on evaluating engagement frameworks, and building the research evidence related to optimal methods for including persons with lived experience in the policy development process, could also assist in building capacity within the health sector to promote knowledge exchange and authentic engagement with persons with lived experience. Partnerships between government departments and organizations that have a strong history of engaging persons with lived experience should be encouraged to reduce the need for departments to reinvent existing infrastructure and programming. Engagement in mental health care reform could also be encouraged by including consumer, caregiver and family member involvement as a criterion for obtaining government funding for mental health related research projects by organizations such as the Canadian Institutes for Health Research.

\section{Conclusion}

In recent years, there has been an historic focus on mental health and mental health care in Canada. Building on this momentum, it is critical that we remain mindful that persons with lived experience of mental health issues should be included and engaged within the policy development process to ensure a recovery-oriented focus. Organizations and government departments must be reflective and creative in designing engagement strategies, as well as look inward to evaluate whether their work environments are really inclusive and supportive of persons with lived experience to bring mental health 'out of the shadows at last. $^{4}$

\section{Acknowledgements}

Amanda Slaunwhite a recipient of the Canadian Institutes for Health Research Frederick Banting and Charles Best Canada Graduate Scholarships Doctoral Award.

\section{References}

1. Church K, Reville D. User involvement in mental health services in Canada: a work in progress. Paper presented at: The International Conference on User Involvement in Mental Health Services; 1988 Sept 26-28; Sussex, England. P. 26.

2. Mental Health Commission of Canada. Changing directions, changing lives: the mental health strategy for Canada. Calgary, Alberta: Mental Health Commission of Canada; 2012. 
3. Partners for Mental Health [Internet]. Ottawa, Ontario: Partners for Mental Health; 2013 [cited 2013 March 8]; Available from: http://www. partnersformh.ca

4. Kirby $\mathrm{M}$, Keon W. Out of the shadows at last: Transforming mental health, mental illness and addictions services in Canada. Standing Senate Committee on Social Affairs, Science and Technology: Government of Canada; 2006.

5. Byrne L, Happell B, Welch T, Moxham LJ. 'Things you can't learn from books': teaching recovery from a lived experience perspective. Int J Ment Health Nurs. 2012 Oct 1; [Epub ahead of print].

6. Tait L, Lester $\mathrm{H}$. Encouraging user involvement in mental health services. Adv Psychiatr Treat. 2005; 11: 168-175.
7. Gregory J. Conceptualising consumer engagement: a review of the literature. Melbourne, Australia: Australian Institute of Health Policy Studies; 2007 Feb. Working Paper 1.

8. Arnstein SR. A ladder of citizen participation. J Am Inst Plann. 1969; 35(4): 216-224.

9. Mental Health Commission of Canada. Toward recovery \& well-being: a framework for a mental health strategy for Canada. Calgary, Alberta: Mental Health Commission of Canada; 2009.

10. Owen D, Ristovski A. A guide to consumer engagement. Tasmania, Australia: Tasmanian Alcohol, Tobacco and Other Drug Treatment Services; 2011.

\section{Amanda Slaunwhite}

Amanda Slaunwhite is a PhD Candidate in the Department of Geography at the University of Victoria, and a Research Affiliate of the Centre for Addictions Research of British Columbia, where she contributes to projects related to mental health and addictions issues, access to treatment, and engagement of persons with lived experience. Amanda's doctoral research focuses on the geographies of alcoholrelated harm and primary care utilization for alcohol-attributed diseases in British Columbia. 